\title{
MEIXNER FUNCTIONS AND POLYNOMIALS RELATED TO LIE ALGEBRA REPRESENTATIONS
}

\author{
WOLTER GROENEVELT AND ERIK KOELINK
}

\begin{abstract}
The decomposition of the tensor product of a positive and a negative discrete series representation of the Lie algebra $\mathfrak{s u}(1,1)$ is a direct integral over the principal unitary series representations. In the decomposition discrete terms can occur, and the discrete terms are a finite number of discrete series representations or one complementary series representation. The interpretation of Meixner functions and polynomials as overlap coefficients in the four classes of representations and the Clebsch-Gordan decomposition, lead to a general bilinear generating function for the Meixner polynomials. Finally, realizing the positive and negative discrete series representations as operators on the spaces of holomorphic and anti-holomorphic functions respectively, a non-symmetric type Poisson kernel is found for the Meixner functions.
\end{abstract}

\section{INTRODUCTION}

The representation theory of Lie algebras is intimately related to special functions of hypergeometric type, see e.g. Vilenkin and Klimyk [18] and references therein. In this paper we consider the Lie algebra $\mathfrak{s u}(1,1)$ spanned by $H, B$ and $C$. In [10, §3] Van der Jeugt and the second author considered the self-adjoint element $X_{a}=-2 a H+B-C, a \geq 0$, in the double and triple tensor product representation of positive discrete series representations, to find convolution identities for orthogonal polynomials, see also [16] where the case $a=1$ is treated. The method used is based on an idea of Granovskii and Zhedanov [1]]; (generalized) eigenvectors of a certain element of the Lie algebra, which acts as a three-term recurrence operator in an irreducible representation of the Lie algebra, can be used to obtain identities for special functions. In this paper we apply this idea to the tensor product of a positive and a negative discrete series representation of $\mathfrak{s u}(1,1)$.

There are four classes of irreducible unitary representations of $\mathfrak{s u}(1,1)$; positive and negative discrete series, principal unitary series and complementary series representations. In the discrete series representations, the overlap coefficients between the standard basis and the basis of eigenvectors of $X_{c}$ are all polynomials; Meixner, Meixner-Pollaczek and Laguerre polynomials, corresponding to $a>1, a<1$ and $a=1$ respectively (see Masson and Repka [13]). This is no longer the case for the principal unitary and the complementary series. Since both these representations appear in the decomposition of the tensor product of a positive and a negative discrete series representation, we have to use non-polynomial functions. In this paper we concentrate on the case $a>1$. Then we need Meixner functions, which are found in [9], [13], using spectral theory of Jacobi operators.

In section 2 we decompose the tensor product of a positive and a negative discrete series representation into a direct integral over the principal series representations. In the decomposition discrete terms can occur, and the discrete terms are a finite number of discrete series representations or one complementary series representation. To find the decomposition, we consider the spectrum of the Casimir operator in the tensor product. As a consequence we find that the continuous dual Hahn polynomials have a natural interpretation as Clebsch-Gordan coefficients for $\mathfrak{s u}(1,1)$.

In section 3 we determine the eigenvectors of $X_{a}$ in the four different representations, as well as the eigenvector of $X_{a}$ in the tensor product representation. Then we determine the Clebsch-Gordan

Date: September 25, 2001. 
coefficients for these eigenvectors, which turn out to be continuous dual Hahn polynomials. Finally we obtain a bilinear generating function for Meixner polynomials.

In section 4 use the holomorphic and anti-holomorphic realization of the positive and negative discrete series representations. This realization was also used in [17] to find generating functions from a tensor product of two positive discrete series representations. Now the eigenvectors of $X_{a}$ become known generating functions for Meixner polynomials. Using the Clebsch-Gordan decomposition from section 2, we obtain a non-symmetric type Poisson kernel for the Meixner functions.

Acknowledgment. We thank Hjalmar Rosengren for useful discussions.

\section{Decomposition of tensor PROduCt REPRESEntations}

In this section we consider the Lie algebra $\mathfrak{s u}(1,1)$ and the four classes of irreducible unitary representations. Then we decompose the tensor product of a positive and a negative discrete series representation into a direct integral of principal unitary series. In the decomposition discrete terms can occur, and the discrete terms are a finite number of discrete series representations or one complementary series representation. We also determine the Clebsch-Gordan coefficients, which turn out to be continuous dual Hahn polynomials.

\subsection{The Lie algebra $\mathfrak{s u}(1,1)$. The Lie algebra $\mathfrak{s u}(1,1)$ is given by}

$$
[H, B]=2 B, \quad[H, C]=-2 C, \quad[B, C]=H .
$$

The $*$-structure is defined by $H^{*}=H$ and $B^{*}=-C$. The Casimir operator $\Omega$ is a central self-adjoint element of the universal enveloping algebra $U(\mathfrak{s u}(1,1))$ and

$$
\Omega=-\frac{1}{4}\left(H^{2}+2 H+4 C B\right)=-\frac{1}{4}\left(H^{2}-2 H+4 B C\right) .
$$

There are four classes of irreducible unitary representations of $\mathfrak{s u}(1,1)$, see [18, §6.4]. The positive discrete series representations $\pi_{k}^{+}$are representations labeled by $k>0$. The representation space is $\ell^{2}\left(\mathbb{Z}_{\geq 0}\right)$ with orthonormal basis $\left\{e_{n}\right\}_{n \in \mathbb{Z}_{\geq 0}}$. The action is given by

$$
\begin{aligned}
\pi_{k}^{+}(H) e_{n} & =2(k+n) e_{n}, \\
\pi_{k}^{+}(B) e_{n} & =\sqrt{(n+1)(2 k+n)} e_{n+1}, \\
\pi_{k}^{+}(C) e_{n} & =-\sqrt{n(2 k+n-1)} e_{n-1}, \\
\pi_{k}^{+}(\Omega) e_{n} & =k(1-k) e_{n} .
\end{aligned}
$$

The negative discrete series representations $\pi_{k}^{-}$are labeled by $k>0$. The representation space is $\ell^{2}\left(\mathbb{Z}_{\geq 0}\right)$ with orthonormal basis $\left\{e_{n}\right\}_{n \in \mathbb{Z}_{\geq 0}}$. The action is given by

$$
\begin{aligned}
\pi_{k}^{-}(H) e_{n} & =-2(k+n) e_{n}, \\
\pi_{k}^{-}(B) e_{n} & =-\sqrt{n(2 k+n-1)} e_{n-1}, \\
\pi_{k}^{-}(C) e_{n} & =\sqrt{(n+1)(2 k+n)} e_{n+1}, \\
\pi_{k}^{-}(\Omega) e_{n} & =k(1-k) e_{n} .
\end{aligned}
$$


The principal series representations $\pi^{\rho, \varepsilon}$ are labeled by $\varepsilon \in[0,1)$ and $\rho \geq 0$, where $(\rho, \varepsilon) \neq\left(0, \frac{1}{2}\right)$. The representation space is $\ell^{2}(\mathbb{Z})$ with orthonormal basis $\left\{e_{n}\right\}_{n \in \mathbb{Z}}$. The action is given by

$$
\begin{aligned}
& \pi^{\rho, \varepsilon}(H) e_{n}=2(\varepsilon+n) e_{n}, \\
& \pi^{\rho, \varepsilon}(B) e_{n}=\sqrt{\left(n+\varepsilon+\frac{1}{2}-i \rho\right)\left(n+\varepsilon+\frac{1}{2}+i \rho\right)} e_{n+1}, \\
& \pi^{\rho, \varepsilon}(C) e_{n}=-\sqrt{\left(n+\varepsilon-\frac{1}{2}-i \rho\right)\left(n+\varepsilon-\frac{1}{2}+i \rho\right)} e_{n-1}, \\
& \pi^{\rho, \varepsilon}(\Omega) e_{n}=\left(\rho^{2}+\frac{1}{4}\right) e_{n} .
\end{aligned}
$$

The complementary series representations $\pi^{\lambda, \varepsilon}$ are labeled by $\varepsilon$ and $\lambda$, where $\varepsilon \in\left[0, \frac{1}{2}\right)$ and $\lambda \in\left(-\frac{1}{2},-\varepsilon\right)$ or $\varepsilon \in\left(\frac{1}{2}, 1\right)$ and $\lambda \in\left(-\frac{1}{2}, \varepsilon-1\right)$. The representation space is $\ell^{2}(\mathbb{Z})$ with orthonormal basis $\left\{e_{n}\right\}_{n \in \mathbb{Z}}$. The action is given by

$$
\begin{aligned}
& \pi^{\lambda, \varepsilon}(H) e_{n}=2(\varepsilon+n) e_{n}, \\
& \pi^{\lambda, \varepsilon}(B) e_{n}=\sqrt{(n+\varepsilon+1+\lambda)(n+\varepsilon-\lambda)} e_{n+1}, \\
& \pi^{\lambda, \varepsilon}(C) e_{n}=-\sqrt{(n+\varepsilon+\lambda)(n+\varepsilon-\lambda-1)} e_{n-1}, \\
& \pi^{\lambda, \varepsilon}(\Omega) e_{n}=-\lambda(1+\lambda) e_{n} .
\end{aligned}
$$

Notice that for $\lambda=-\frac{1}{2}+i \rho$ the actions in the principal series and in the complementary series are the same.

The operators are unbounded, defined on the space of finite linear combinations of the basisvectors. From general theory, see Schmüdgen [15, Ch.8, Ch.10], it follows that all operators are closable.

2.2. Tensor products of positive and negative discrete series representations. In order to decompose the tensor product of a positive and a negative discrete series representation we need the continuous dual Hahn polynomials, which are a special case of the Wilson polynomials, see Wilson [20, §4]; see also [2, §6.10], [8];

$$
S_{n}(y ; a, b, c)=(a+b)_{n}(a+c)_{n 3} F_{2}\left(\begin{array}{c}
-n, a+i x, a-i x \\
a+b, a+c
\end{array} ; 1\right), \quad x^{2}=y .
$$

For real parameters $a, b, c$, with $a+b, a+c, b+c$ positive, the continuous dual Hahn polynomials are orthogonal with respect to a positive measure, supported on a subset of $\mathbb{R}$.

The orthonormal continuous dual Hahn polynomials

$$
\hat{S}_{n}(y)=\hat{S}_{n}(y ; a, b, c)=\frac{(-1)^{n} S_{n}(y ; a, b, c)}{\sqrt{n !(a+b)_{n}(a+c)_{n}(b+c)_{n}}}
$$

satisfy the three-term recurrence relation

$$
y \hat{S}_{n}(y)=a_{n} \hat{S}_{n+1}(y)+b_{n} \hat{S}_{n}(y)+a_{n-1} \hat{S}_{n-1}(y)
$$

where

$$
\begin{aligned}
& a_{n}=\sqrt{(n+1)(n+a+b)(n+a+c)(n+b+c)}, \\
& b_{n}=2 n^{2}+2 n\left(a+b+c-\frac{1}{2}\right)+a b+a c+b c .
\end{aligned}
$$

By Kummer's transformation, see e.g. [2, Cor. 3.3.5], the polynomials $S_{n}$ and $\hat{S}_{n}$ are symmetric in $a, b$ and $c$. 
Without loss of generality we assume that $a$ is the smallest of the real parameters $a, b$ and $c$. Let $d \mu^{2}(\cdot ; a, b, c)$ be the measure defined by

$$
\begin{gathered}
\int_{\mathbb{R}} f(y) d \mu^{2}(y ; a, b, c)=\frac{1}{2 \pi} \int_{0}^{\infty}\left|\frac{\Gamma(a+i x) \Gamma(b+i x) \Gamma(c+i x)}{\Gamma(2 i x)}\right|^{2} \frac{f\left(x^{2}\right)}{\Gamma(a+b) \Gamma(a+c) \Gamma(b+c)} d x \\
+\frac{\Gamma(b-a) \Gamma(c-a)}{\Gamma(-2 a) \Gamma(b+c)} \sum_{k=0}^{K}(-1)^{k} \frac{(2 a)_{k}(a+1)_{k}(a+b)_{k}(a+c)_{k}}{(a)_{k}(a-b+1)_{k}(a-c+1)_{k} k !} f\left(-(a+k)^{2}\right),
\end{gathered}
$$

where $K$ is the largest non-negative integer such that $a+K<0$. In particular, the measure $d \mu^{2}(\cdot ; a, b, c)$ is absolutely continuous if $a \geq 0$. The measure is positive under the conditions $a+b>0, a+c>0$ and $b+c>0$. Then the polynomials $\hat{S}_{n}(y ; a, b, c)$ are orthonormal with respect to the measure $d \mu^{2}(y ; a, b, c)$.

Later on we also use the notation $d \mu(\cdot ; a, b, c)$, by this we denote the measure defined by

$$
\begin{gathered}
\int_{\mathbb{R}} f(y) d \mu(y ; a, b, c)=\frac{1}{\sqrt{2 \pi}} \int_{0}^{\infty}\left|\frac{\Gamma(a+i x) \Gamma(b+i x) \Gamma(c+i x)}{\Gamma(2 i x)}\right| \frac{f\left(x^{2}\right)}{\sqrt{\Gamma(a+b) \Gamma(a+c) \Gamma(b+c)}} d x \\
+\sqrt{\frac{\Gamma(b-a) \Gamma(c-a)}{\Gamma(-2 a) \Gamma(b+c)}} \sum_{k=0}^{K} \sqrt{(-1)^{k} \frac{(2 a)_{k}(a+1)_{k}(a+b)_{k}(a+c)_{k}}{(a)_{k}(a-b+1)_{k}(a-c+1)_{k} k !}} f\left(-(a+k)^{2}\right) .
\end{gathered}
$$

Since $d \mu^{2}(\cdot ; a, b, c)$ is a positive measure for $a+b>0, a+c>0$ and $b+c>0$, the measure $d \mu(\cdot ; a, b, c)$ is a positive measure.

In order to determine the decomposition of the tensor product representation $\pi_{k_{1}}^{+} \otimes \pi_{k_{2}}^{-}$, we calculate the action of the Casimir operator $\Omega$. In the tensor product, we need the comultiplication $\Delta$. Recall that for an element $Y$ of the Lie algebra $\mathfrak{s u}(1,1)$, we have $\Delta(Y)=1 \otimes Y+Y \otimes 1$ and $\Delta$ is extended to $U(\mathfrak{s u}(1,1))$ as an algebra homomorphism. Then from (2.1) we obtain

$$
\Delta(\Omega)=1 \otimes \Omega+\Omega \otimes 1-\frac{1}{2} H \otimes H-(C \otimes B+B \otimes C) .
$$

We use this expression, (2.2) and (2.3), to calculate the action of $\Omega$ in the tensor product

$$
\begin{aligned}
\pi_{k_{1}}^{+} \otimes \pi_{k_{2}}^{-}(\Delta(\Omega)) & e_{n} \otimes e_{m}= \\
& -\sqrt{(n+1)\left(2 k_{1}+n\right)(m+1)\left(2 k_{2}+m\right)} e_{n+1} \otimes e_{m+1} \\
& +\left(k_{1}\left(1-k_{1}\right)+k_{2}\left(1-k_{2}\right)+2\left(k_{1}+n\right)\left(k_{2}+m\right)\right) e_{n} \otimes e_{m} \\
& -\sqrt{n\left(2 k_{1}+n-1\right) m\left(2 k_{2}+m-1\right)} e_{n-1} \otimes e_{m-1},
\end{aligned}
$$

We see that $\pi_{k_{1}}^{+} \otimes \pi_{k_{2}}^{-}(\Delta(\Omega))$ is a three-term recurrence operator, which we can identify with the three-term recurrence relation for the continuous dual Hahn polynomials. In order to do so we define for $p \in \mathbb{Z}$ and $n \in \mathbb{Z}_{\geq 0}$, elements $f_{n}^{p} \in \ell^{2}\left(\mathbb{Z}_{\geq 0}\right) \otimes \ell^{2}\left(\mathbb{Z}_{\geq 0}\right)$ by

$$
f_{n}^{p}= \begin{cases}(-1)^{n} e_{n} \otimes e_{n-p}, & p \leq 0, \\ (-1)^{n} e_{n+p} \otimes e_{n}, & p \geq 0 .\end{cases}
$$

Note that $\left\{f_{n}^{p}\right\}_{n \in \mathbb{Z}_{\geq 0}, p \in \mathbb{Z}}$ is an orthonormal basis for $\ell^{2}\left(\mathbb{Z}_{\geq 0}\right) \otimes \ell^{2}\left(\mathbb{Z}_{\geq 0}\right)$. For fixed $p$ define the space

$$
H_{p}=\overline{\mathbb{C}\left\{f_{n}^{p} \mid n \in \mathbb{Z}_{\geq 0}\right\}},
$$

then $\pi_{k_{1}}^{+} \otimes \pi_{k_{2}}^{-}(\Delta(\Omega))$ is an unbounded symmetric tridiagonal operator on $H_{p}$ with domain the space of finite linear combinations of $f_{n}^{p}$. 
We define operators $\Lambda_{p}: H_{p} \rightarrow L^{2}\left(\mathbb{R}, d \mu^{2}(y ; p)\right)$ by

$$
f_{n}^{p} \mapsto \begin{cases}\hat{S}_{n}\left(y ; k_{1}-k_{2}+\frac{1}{2}, k_{1}+k_{2}-\frac{1}{2}, k_{2}-k_{1}-p+\frac{1}{2}\right), & p \leq 0 \\ \hat{S}_{n}\left(y ; k_{2}-k_{1}+\frac{1}{2}, k_{1}+k_{2}-\frac{1}{2}, k_{1}-k_{2}+p+\frac{1}{2}\right), & p \geq 0\end{cases}
$$

where $d \mu^{2}(y ; p)$ denotes the measure $d \mu^{2}(y ; a, b, c)$ with

$$
a=\left\{\begin{array}{ll}
k_{1}-k_{2}+\frac{1}{2}, & p \leq 0, \\
k_{2}-k_{1}+\frac{1}{2}, & p \geq 0,
\end{array} \quad b=k_{1}+k_{2}-\frac{1}{2}, \quad c= \begin{cases}k_{2}-k_{1}-p+\frac{1}{2}, & p \leq 0 \\
k_{1}-k_{2}+p+\frac{1}{2}, & p \geq 0\end{cases}\right.
$$

Proposition 2.1. For every $p \in \mathbb{Z}$ the operator $\Lambda_{p}$ is unitary and intertwines $\pi_{k_{1}}^{+} \otimes \pi_{k_{2}}^{-}(\Delta(\Omega))$ acting on $H_{p}$, with $M_{y+\frac{1}{4}}$ acting on $L^{2}\left(\mathbb{R}, d \mu^{2}(y ; p)\right)$.

Here $M_{g}$ denotes multiplication by the function $g$, so $M_{g} f(y)=g(y) f(y)$.

Proof. Letting $\pi_{k_{1}}^{+} \otimes \pi_{k_{2}}^{-}(\Delta(\Omega))$ act on an element $f_{n}^{p}$, we see that $\left.\pi_{k_{1}}^{+} \otimes \pi_{k_{2}}^{-}(\Delta(\Omega))\right|_{H_{p}}$ is an unbounded Jacobi operator, see Akhiezer [1]. Comparing the coefficients with the three-term recurrence relation (2.7) for the continuous dual Hahn polynomials, we see that we can identify $\left.\pi_{k_{1}}^{+} \otimes \pi_{k_{2}}^{-}(\Delta(\Omega))\right|_{H_{p}}-\frac{1}{4}$ with (2.7) using (2.8) by a straightforward computation. Since the corresponding moment problem is determinate, $\left.\pi_{k_{1}}^{+} \otimes \pi_{k_{2}}^{-}(\Delta(\Omega))\right|_{H_{p}}$ is essentially self-adjoint and the operator $\Lambda_{p}$ intertwines $\left.\pi_{k_{1}}^{+} \otimes \pi_{k_{2}}^{-}(\Delta(\Omega))\right|_{H_{p}}$ with $M_{y+\frac{1}{4}}$. Since the continuous dual Hahn polynomials $\hat{S}_{n}$ are dense in $L^{2}\left(\mathbb{R}, d \mu^{2}(y ; p)\right), \Lambda_{p}$ maps one orthonormal basis onto another. Therefore $\Lambda_{p}$ is a unitary operator.

Using proposition 2.1 we can find the spectrum of $\pi_{k_{1}}^{+} \otimes \pi_{k_{2}}^{-}(\Delta(\Omega))$, which is an essentially selfadjoint operator on $\ell^{2}\left(\mathbb{Z}_{\geq 0}\right) \otimes \ell^{2}\left(\mathbb{Z}_{\geq 0}\right)$. From the orthonormality measure for the continuous dual Hahn polynomials, we see that the spectrum has a continuous part $\left[\frac{1}{4}, \infty\right)$ and a finite discrete part, depending on the parameters $k_{1}$ and $k_{2}$. From now on we assume that $k_{1} \leq k_{2}$.

For the continuous part of the spectrum, we recognize the action of $\Omega$ in the principal series representation, using $y=\rho^{2}$. From the action of $H$ in the tensor product, we obtain $\varepsilon=k_{1}-k_{2}+L$, where $L$ is the unique non-negative integer such that $\varepsilon \in[0,1)$.

If $k_{1}+k_{2}<\frac{1}{2}$ the point spectrum contains one term: $\frac{1}{4}-\left(k_{1}+k_{2}-\frac{1}{2}\right)^{2}$. Using $\lambda=-k_{1}-k_{2}$ we recognize here the action of $\Omega$ in the complementary series representation labeled by $\lambda$ and $\varepsilon$.

For $k_{1}-k_{2}<-\frac{1}{2}$ the spectrum has $K+1$ discrete terms for $p \leq 0$ and $K+1-p$ terms for $0 \leq p \leq$ $K$. If $p>K$ there is no discrete part. Here $K$ is the largest integer such that $k_{1}-k_{2}+\frac{1}{2}+K<0$. All the terms in the discrete part of the spectrum have the form $\frac{1}{4}-\left(k_{1}-k_{2}+\frac{1}{2}+j\right)^{2}, j=0,1, \ldots, K$. From this we recognize the action of $\Omega$ in a discrete series representation $\pi_{k_{2}-k_{1}-j}$. From the action of $H$, we find that this is a negative discrete series representation.

Now we can prove the following theorem. 
Theorem 2.2. For $k_{1} \leq k_{2}$ the decomposition of the tensor product of positive and negative discrete series representations of $\mathfrak{s u}(1,1)$ is

$$
\begin{array}{rlr}
\pi_{k_{1}}^{+} \otimes \pi_{k_{2}}^{-} \cong \int_{0}^{\infty} \pi^{\rho, \varepsilon} d \rho, & k_{1}-k_{2} \geq-\frac{1}{2}, k_{1}+k_{2} \geq \frac{1}{2}, \\
\pi_{k_{1}}^{+} \otimes \pi_{k_{2}}^{-} \cong \int_{0}^{\infty} \pi^{\rho, \varepsilon} d \rho \oplus \pi^{\lambda, \varepsilon}, & k_{1}+k_{2}<\frac{1}{2}, \\
\pi_{k_{1}}^{+} \otimes \pi_{k_{2}}^{-} \cong \int_{0}^{\infty} \pi^{\rho, \varepsilon} d \rho \oplus \bigoplus_{j \in \mathbb{Z}_{\geq 0}} \pi_{k_{2}-k_{1}-j}^{-}, & k_{1}-k_{2}<-\frac{1}{2},
\end{array}
$$

where $\varepsilon=k_{1}-k_{2}+L, L$ is the unique integer such that $\varepsilon \in[0,1)$ and $\lambda=-k_{1}-k_{2}$. Further, under the identification above,

$$
e_{n_{1}} \otimes e_{n_{2}}=(-1)^{n_{2}} \int_{\mathbb{R}} \hat{S}_{n}\left(y ; n_{1}-n_{2}\right) e_{n_{1}-n_{2}-L} d \mu\left(y ; n_{1}-n_{2}\right),
$$

where $n=\min \left\{n_{1}, n_{2}\right\}, \hat{S}_{n}(y ; p)$ is a continuous dual Hahn polynomial,

$$
\hat{S}_{n}(y ; p)= \begin{cases}\hat{S}_{n}\left(y ; k_{1}-k_{2}+\frac{1}{2}, k_{1}+k_{2}-\frac{1}{2}, k_{2}-k_{1}-p+\frac{1}{2}\right), & p \leq 0, \\ \hat{S}_{n}\left(y ; k_{2}-k_{1}+\frac{1}{2}, k_{1}+k_{2}-\frac{1}{2}, k_{1}-k_{2}+p+\frac{1}{2}\right), & p \geq 0,\end{cases}
$$

and

$$
d \mu(y ; p)= \begin{cases}d \mu\left(y ; k_{1}-k_{2}+\frac{1}{2}, k_{1}+k_{2}-\frac{1}{2}, k_{2}-k_{1}-p+\frac{1}{2}\right), & p \leq 0, \\ d \mu\left(y ; k_{2}-k_{1}+\frac{1}{2}, k_{1}+k_{2}-\frac{1}{2}, k_{1}-k_{2}+p+\frac{1}{2}\right), & p \geq 0 .\end{cases}
$$

So the continuous dual Hahn polynomials have an interpretation as Clebsch-Gordan coefficients for $\mathfrak{s u}(1,1)$. The inversion of (2.9) can be given explicitly, e.g. for an element

$$
f \otimes e_{r-L}=\int_{0}^{\infty} f(x) e_{r-L} d x \in L^{2}(0, \infty) \otimes \ell^{2}(\mathbb{Z}) \cong \int_{0}^{\infty} \ell^{2}(\mathbb{Z}) d x
$$

in the representation space of the direct integral, we have

$$
f \otimes e_{r-L}= \begin{cases}\sum_{p=0}^{\infty}(-1)^{p-r}\left[\int_{\mathbb{R}} \hat{S}_{p}(y ; r) f(y) d \mu(y ; r)\right] e_{p} \otimes e_{p-r}, & r \leq 0, \\ \sum_{p=0}^{\infty}(-1)^{p}\left[\int_{\mathbb{R}} \hat{S}_{p}(y ; r) f(y) d \mu(y ; r)\right] e_{p+r} \otimes e_{p}, & r \geq 0 .\end{cases}
$$

For the discrete components in theorem 2.2 we can replace $f$ by a Dirac delta function at the appropriate points of the discrete mass of $d \mu(\cdot ; r)$.

Proof. First we work out the proof for $k_{1}-k_{2} \geq-\frac{1}{2}$ and $k_{1}+k_{2} \geq \frac{1}{2}$, so $d \mu\left(y ; n_{1}-n_{2}\right)$ only has a absolutely continuous part. We denote the continuous part of the measure $d \mu(y ; a, b, c)$ by $W(y ; a, b, c)$, so

$$
W(y ; a, b, c)=\left|\frac{\Gamma(a+i x) \Gamma(b+i x) \Gamma(c+i x)}{\Gamma(2 i x)}\right| \frac{1}{\sqrt{2 \pi \Gamma(a+b) \Gamma(a+c) \Gamma(b+c)}}, \quad y=x^{2} .
$$


Observe that

$$
\frac{W(y ; a, b, c+1)}{W(y ; a, b, c)}=\sqrt{\frac{c^{2}+y}{(a+c)(b+c)}} .
$$

Define an operator $\Lambda$ by gluing together all the operators $\Lambda_{p}, p \in \mathbb{Z}$,

$$
\Lambda\left(e_{n_{1}} \otimes e_{n_{2}}\right)=(-1)^{n_{2}} \int_{0}^{\infty} \hat{S}_{l}\left(x^{2} ; n_{1}-n_{2}\right) W\left(x^{2} ; p\right) e_{n_{1}-n_{2}-L} d x,
$$

where $l=\min \left\{n_{1}, n_{2}\right\}$ and $e_{n_{1}-n_{2}-L}$ inside the integral is an orthonormal basisvector of the representation space for the principal series representation $\pi^{x, \varepsilon}$. Note that $\Lambda$ is unitary.

We use (2.2) and (2.3) to find the action of $B$ in the tensor product for $n_{1}=n$ and $n_{1}-n_{2}=p<0$;

$$
\begin{aligned}
\Lambda & \left(\pi_{k_{1}}^{+} \otimes \pi_{k_{2}}^{-}(\Delta(B)) e_{n} \otimes e_{n-p}\right) \\
= & (-1)^{n-p} \int_{0}^{\infty} \sqrt{(n+1)\left(2 k_{1}+n\right)} \hat{S}_{n+1}\left(x^{2} ; p+1\right) W\left(x^{2} ; p+1\right) e_{p-L+1} d x \\
& -(-1)^{n-p-1} \int_{0}^{\infty} \sqrt{(n-p)\left(2 k_{2}+n-p-1\right)} \hat{S}_{n}\left(x^{2} ; p+1\right) W\left(x^{2} ; p+1\right) e_{p-L+1} d x \\
= & (-1)^{n-p} \int_{0}^{\infty} \sqrt{\left(p-L+k_{1}-k_{2}+L+\frac{1}{2}\right)^{2}+x^{2}} \hat{S}_{n}\left(x^{2} ; p\right) W\left(x^{2} ; p\right) e_{p-L+1} d x \\
= & \int_{0}^{\infty} \pi^{x, \varepsilon}(B) d x \circ \Lambda\left(e_{n} \otimes e_{n-p}\right),
\end{aligned}
$$

where $\pi^{x, \varepsilon}$ denotes the principal series representation. Here we use (2.11) and the following contiguous relation

$$
\begin{aligned}
& \frac{c^{2}+y}{\sqrt{(a+c)(b+c)}} \hat{S}_{n}(y ; a, b, c+1)= \\
& \sqrt{(n+1)(a+b+n)} \hat{S}_{n+1}(y ; a, b, c)+\sqrt{(a+c+n)(b+c+n)} \hat{S}_{n}(y ; a, b, c) .
\end{aligned}
$$

This relation can be verified by expanding

$$
\left(y+c^{2}\right) \hat{S}_{n}(y ; a, b, c+1)=\sum_{i=0}^{n+1} c_{i} \hat{S}_{i}(y ; a, b, c),
$$

where

$$
c_{i}=\int_{\mathbb{R}}\left(y+c^{2}\right) \hat{S}_{n}(y ; a, b, c+1) \hat{S}_{i}(y ; a, b, c) d \mu^{2}(y ; a, b, c) .
$$

From (2.11) and the orthogonality relations for $\hat{S}_{n}(y ; a, b, c+1)$ we see that $c_{i}=0$ for $i<n$. The coefficients $c_{n}$ and $c_{n+1}$ follow easily from the integral and the leading coefficient of $\hat{S}_{n}(y ; a, b, c)=$ $\left(\sqrt{n !(a+b)_{n}(a+c)_{n}(b+c)_{n}}\right)^{-1} y^{n}+\ldots$

For the case $p \geq 0$ the intertwining property for the action of $B$ follows similarly using the contiguous relation

$$
\begin{aligned}
& \sqrt{(a+c-1)(b+c-1)} \hat{S}_{n}(y ; a, b, c-1)= \\
& \sqrt{(n+a+c-1)(n+b+c-1)} \hat{S}_{n}(y ; a, b, c)+\sqrt{n(n+a+b-1)} \hat{S}_{n-1}(y ; a, b, c) .
\end{aligned}
$$

This companion formula to (2.13) is checked in the same way as (2.13). 
So we now have shown

$$
\Lambda \circ \pi_{k_{1}}^{+} \otimes \pi_{k_{2}}^{-}(\Delta(Y))=\int_{0}^{\infty} \pi^{\rho, \varepsilon}(Y) d \rho \circ \Lambda,
$$

for $Y=B$. The case $Y=H$ of (2.14) is straightforward from (2.2), (2.3), (2.4) and (2.12). The case $Y=C$ of (2.14) can be deduced as for $Y=B$, or it can be deduced from the case $Y=B$ and the unitary properties of $\Lambda$ and the representations of $\mathfrak{s u}(1,1)$.

We now have proved the theorem for the case $k_{1}-k_{2} \geq-\frac{1}{2}, k_{1}+k_{2} \geq \frac{1}{2}$. The other cases can be proved in the same way, only some more bookkeeping is involved for the discrete mass points.

Remark 2.3. (i) For $k_{1}+k_{2}<\frac{1}{2}$ a complementary series representation enters the decomposition. This fact has already been observed by Ørsted and Zhang in [14, §3] for the case $k_{1}=k_{2}$. They also found continuous dual Hahn polynomials as Clebsch-Gordan coefficients. For the group $S U(1,1)$ there is no complementary series representation in the decomposition of the tensor product, since then $k_{1}, k_{2} \in \frac{1}{2} \mathbb{N}$. For the group case we refer to Kerimov and Verdiyev [7], but they do not mention that the Clebsch-Gordan coefficients are continuous dual Hahn polynomials. For $k_{1}=k_{2}$ the tensor product decomposition naturally occurs in the framework of canonical representations, see Hille [5] and references therein.

(ii) For $k_{1}-k_{2}<-\frac{1}{2}$, the measure on the right hand side of (2.9) has only a discrete part for $n_{1}-n_{2} \leq K$.

(iii) The representation on the right hand side in theorem 2.2 are defined on the dense domain consisting of finite linear combinations of elements as on the right hand side of (2.9). Then $\Lambda$ is an intertwiner in the sense of [15, §8.2].

\section{Coupled and uncoupled eigenvectors of $X_{c}$}

In this section we consider a self-adjoint element $X_{c}$ in $\mathfrak{s u}(1,1)$. We determine eigenvectors of this element in the four different representations. Then we determine the Clebsch-Gordan coefficients for this basis of eigenvectors in the tensor product. As a result we find a general bilinear generating function for Meixner polynomials.

3.1. Eigenvectors of $X_{c}$ in irreducible representations. The Meixner polynomials (see [2], [8]) are defined by

$$
M_{n}(x ; \beta, c)={ }_{2} F_{1}\left(\begin{array}{c}
-n,-x \\
\beta
\end{array} ; 1-\frac{1}{c}\right) .
$$

For $\beta>0$ and $0<c<1$, these are orthogonal polynomials with respect to a positive measure on $\mathbb{Z}_{\geq 0}$. The orthonormal Meixner polynomials

$$
\hat{M}_{n}(x)=\hat{M}_{n}(x ; \beta, c)=\sqrt{\frac{(\beta)_{n} c^{n}}{n !}} M_{n}(x ; \beta, c)
$$

satisfy the following three-term recurrence relation

$$
\begin{aligned}
\frac{(c-1)\left(x+\frac{1}{2} \beta\right)}{\sqrt{c}} \hat{M}_{n}(x)= & \sqrt{(n+1)(n+\beta)} \hat{M}_{n+1}(x)+ \\
& -\frac{(c+1)\left(n+\frac{1}{2} \beta\right)}{\sqrt{c}} \hat{M}_{n}(x)+\sqrt{n(n-1+\beta)} \hat{M}_{n-1} .
\end{aligned}
$$

Define the weight function $w(x ; \beta, c)$ by

$$
w(x ; \beta, c)=\frac{(\beta)_{x}}{x !} c^{x}(1-c)^{\beta},
$$


then the Meixner polynomials are orthonormal in $x$ with respect to this weight function;

$$
\sum_{x=0}^{\infty} \hat{M}_{n}(x) \hat{M}_{m}(x) w(x ; \beta, c)=\delta_{m n} .
$$

From the definition it is clear that the Meixner polynomials are self-dual, i.e. invariant under interchanging $n$ and $x$. This gives us another orthogonality relation

$$
\sum_{n=0}^{\infty} \hat{M}_{n}(x) \hat{M}_{n}(y) w(x ; \beta, c)=\delta_{x y} .
$$

The Meixner functions (see Masson and Repka [13, §3] and Koelink [9, §4.4]) are defined by

$$
\begin{aligned}
\hat{m}_{n}(x)=\hat{m}_{n}(x ; \lambda, \varepsilon, c)=\left(\frac{1-c}{\sqrt{c}}\right)^{-n} & \frac{\sqrt{\Gamma(n+\lambda+\varepsilon+1) \Gamma(n+\varepsilon-\lambda)}}{\Gamma(n+1-x)} \\
& \times{ }_{2} F_{1}\left(\begin{array}{c}
n+\varepsilon+\lambda+1, n+\varepsilon-\lambda \\
n+1-x
\end{array} ; \frac{c}{c-1}\right) .
\end{aligned}
$$

Here we use the analytic continuation to $\mathbb{C} \backslash[1, \infty)$ of the ${ }_{2} F_{1}$-series. The Meixner functions are orthonormal with respect to a positive measure on $\mathbb{Z}$, if $0<c<1,0 \leq \varepsilon<1$ and $-\frac{1}{2}<\lambda<-\varepsilon$ or $-\frac{1}{2}<\lambda<\varepsilon-1$ or $\lambda=-\frac{1}{2}+i \rho, \rho \geq 0$. If $n+\lambda+\varepsilon+1$ or $n+\varepsilon-\lambda$ is a non-positive integer, the ${ }_{2} F_{1}$-series terminates and can be written as a Meixner polynomial. The Meixner funcions satisfy the following three-term recurrence relation

$$
\begin{aligned}
\frac{(c-1)(\varepsilon+x)}{\sqrt{c}} \hat{m}_{n}(x)= & \sqrt{(n+\varepsilon+\lambda+1)(n+\varepsilon-\lambda)} \hat{m}_{n+1}(x)+ \\
& -\frac{(1+c)(n+\varepsilon)}{\sqrt{c}} \hat{m}_{n}(x)+\sqrt{(n+\varepsilon+\lambda)(n+\varepsilon-\lambda-1)} \hat{m}_{n-1}(x) .
\end{aligned}
$$

Define the weight function $\widetilde{w}(x ; \lambda, \varepsilon, c)$ by

$$
\widetilde{w}(x ; \lambda, \varepsilon, c)=\frac{c^{-x}(1-c)^{-2 \varepsilon}}{\Gamma(\varepsilon+x-\lambda) \Gamma(\varepsilon+\lambda+x+1)},
$$

then the Meixner functions are orthonormal with respect to this weight function

$$
\sum_{x=-\infty}^{\infty} \hat{m}_{m}(x) \hat{m}_{n}(x) \widetilde{w}(x ; \lambda, \varepsilon, c)=\delta_{m n} .
$$

The Meixner functions also satisfy the dual orthonormality relation

$$
\sum_{n=-\infty}^{\infty} \hat{m}_{n}(x) \hat{m}_{n}(y) \widetilde{w}(x ; \lambda, \varepsilon, c)=\delta_{x y} .
$$

Note that for $\lambda=-\frac{1}{2}+i \rho$, the ${ }_{2} F_{1}$-series in the definition of the Meixner function is a Jacobi function $\varphi_{2 \rho}^{(n-x, n+x+2 \varepsilon)}(c /(1-c))$, where the Jacobi function is defined by

$$
\varphi_{\sigma}^{(\alpha, \beta)}(t)={ }_{2} F_{1}\left(\begin{array}{c}
\frac{1}{2}(\alpha+\beta+1-i \sigma), \frac{1}{2}(\alpha+\beta+1+i \sigma) \\
\alpha+1
\end{array} ;-t\right) .
$$

For Jacobi functions, see [12] or [18, §7.4.3].

We define the self-adjoint element $X_{c}$ in $\mathfrak{s u}(1,1)$ by

$$
X_{c}=-\frac{1+c}{2 \sqrt{c}} H+B-C .
$$

We first consider the action of $X_{c}$ in the positive and negative discrete series representations. 
Proposition 3.1. For $x \in \mathbb{Z}_{\geq 0}$

$$
v_{x}^{+}=\sum_{n=0}^{\infty} \hat{M}_{n}(x ; 2 k, c) \sqrt{w(x ; 2 k, c)} e_{n} \in \ell^{2}\left(\mathbb{Z}_{\geq 0}\right),
$$

is a unit eigenvector of $\pi_{k}^{+}\left(X_{c}\right)$ for the eigenvalue $(c-1)(x+k) / \sqrt{c}$. And for $x \in \mathbb{Z}_{\geq 0}$

$$
v_{x}^{-}=\sum_{n=0}^{\infty} \hat{M}_{n}(x ; 2 k, c) \sqrt{w(x ; 2 k, c)} e_{n} \in \ell^{2}\left(\mathbb{Z}_{\geq 0}\right)
$$

is a unit eigenvector of $\pi_{k}^{-}\left(X_{c}\right)$ for the eigenvalue $-(c-1)(x+k) / \sqrt{c}$. Moreover, $\left\{v_{x}^{\bullet}\right\}_{x \in \mathbb{Z}_{\geq 0}}$, $\bullet=+,-$, is an orthonormal basis of $\ell^{2}\left(\mathbb{Z}_{\geq 0}\right)$.

For the positive discrete series this is $[10$, prop. 3.1]), and for the negative discrete series this proceeds completely analogously. Proposition 3.1 also shows that $\pi_{k}^{+}\left(X_{c}\right)$, respectively $\pi_{k}^{-}\left(X_{c}\right)$, is essentially self-adjoint, see [1]. The self-adjoint extension is defined by the same formula on its maximal domain, and the domain $\mathcal{D}$ of the self-adjoint extension can be discribed in terms of the eigenvectors of proposition 3.1 as

$$
\mathcal{D}=\left\{\sum_{x \in \mathbb{Z}_{\geq 0}} c_{x} v_{x}^{+} \in \ell^{2}\left(\mathbb{Z}_{\geq 0}\right) \mid \sum_{x \in \mathbb{Z}_{\geq 0}} c_{x}(x+k) v_{x}^{+} \in \ell^{2}\left(\mathbb{Z}_{\geq 0}\right)\right\},
$$

and similarly for the self-adjoint extension of $\pi_{k}^{-}\left(X_{c}\right)$.

Next we consider the action of $X_{c}$ in the principal and complementary series representations. We denote both these representations here by $\pi^{\lambda, \varepsilon}$, where $\lambda=-\frac{1}{2}+i \rho, \rho \geq 0$, in the principal series.

Proposition 3.2. For $x \in \mathbb{Z}$

$$
w_{x}^{\lambda, \varepsilon}=\sum_{n=-\infty}^{\infty} \hat{m}_{n}(x ; \lambda, \varepsilon, c) \sqrt{\widetilde{w}(x ; \lambda, \varepsilon, c)} e_{n} \in \ell^{2}(\mathbb{Z})
$$

is a unit eigenvector of $\pi^{\lambda, \varepsilon}\left(X_{c}\right)$ for the eigenvalue $(c-1)(\varepsilon+x) / \sqrt{c}$. Moreover, $\left\{w_{x}^{\lambda, \varepsilon}\right\}_{x \in \mathbb{Z}}$ is an orthonormal basis of $\ell^{2}(\mathbb{Z})$.

Proof. The proof follows using a doubly infinite Jacobi operator, corresponding to (3.5) and its spectral decomposition given by (3.6), see [9], 13.

Remark 3.3. Note that the spectrum of $\pi^{\lambda, \varepsilon}\left(X_{c}\right)$ is independent of $\lambda$.

We will denote the eigenvector $w_{x}^{\lambda, \varepsilon}$ for the principal series, by $v_{x}^{\rho, \varepsilon}$, so $v_{x}^{\rho, \varepsilon}=w_{x}^{-\frac{1}{2}+i \rho, \varepsilon}$.

Proposition 3.2 implies that $\pi^{\lambda, \varepsilon}\left(X_{c}\right)$ is essentially self-adjoint, see [9], [13], and that its selfadjoint extension is given by the same formula on its maximal domain. In terms of the eigenvectors of proposition 3.2 the domain is

$$
\mathcal{D}=\left\{\sum_{x \in \mathbb{Z}} c_{x} w_{x}^{\lambda, \varepsilon} \in \ell^{2}(\mathbb{Z}) \mid \sum_{x \in \mathbb{Z}} c_{x}(\varepsilon+x) w_{x}^{\lambda, \varepsilon} \in \ell^{2}(\mathbb{Z})\right\} .
$$

3.2. Eigenvectors of $X_{c}$ in the tensor product. In this subsection we want to link the coupled and uncoupled eigenvectors of $X_{c}$ in the tensor product $\pi_{k_{1}}^{+} \otimes \pi_{k_{2}}^{-}$, and calculate the corresponding Clebsch-Gordan coefficients. We discuss the method that reduces the problem to theorem 2.2 and then indicate another method, based on the Jacobi transform, in remark 3.5. 
We define elements $H_{c}, B_{c}$ and $C_{c}$ by

$$
\begin{aligned}
H_{c} & =\frac{1+c}{1-c} H-\frac{2 \sqrt{c}}{1-c} B+\frac{2 \sqrt{c}}{1-c} C, \\
B_{c} & =-\frac{\sqrt{c}}{1-c} H+\frac{1}{1-c} B-\frac{c}{1-c} C, \\
C_{c} & =\frac{\sqrt{c}}{1-c} H-\frac{c}{1-c} B+\frac{1}{1-c} C .
\end{aligned}
$$

then $H_{c}=g H g^{-1}, B_{c}=g B g^{-1}$ and $C_{c}=g C g^{-1}$ with $g \in S U(1,1), g=\left(\begin{array}{ll}a & b \\ b & a\end{array}\right), a=1 / \sqrt{1-c}$, $b=\sqrt{c /(1-c)}$. Then the relations

$$
\left[H_{c}, B_{c}\right]=2 B_{c}, \quad\left[H_{c}, C_{c}\right]=-2 C_{c}, \quad\left[B_{c}, C_{c}\right]=H_{c}
$$

follow, and also

$$
H_{c}^{*}=H_{c}, \quad B_{c}^{*}=-C_{c} .
$$

Note that $H_{c}=\frac{2 \sqrt{c}}{c-1} X_{c}$, so that $H_{c}$ act diagonally with respect to orthonormal bases in propositions 3.1 and 3.2 in all representations under consideration. Moreover, $\Omega=-\frac{1}{4}\left(H_{c}^{2}+2 H_{c}+4 C_{c} B_{c}\right)$ by this argument, or by a straightforward computation.

Next we want to calculate the actions of $\pi_{k}^{+}\left(B_{c}\right), \pi_{k}^{-}\left(B_{c}\right), \pi^{\lambda, \varepsilon}\left(B_{c}\right)$ on the eigenvectors of $\pi_{k}^{+}\left(H_{c}\right)$, $\pi_{k}^{-}\left(H_{c}\right), \pi^{\lambda, \varepsilon}\left(H_{c}\right)$ of propositions 3.1 and 3.2 . From $-4 \Omega=H_{c}^{2}+2 H_{c}+4 C_{c} B_{c}, B_{c}^{*}=-C_{c}$, using the polar decomposition for the closure of $\pi_{k}^{+}\left(B_{c}\right)$, we see that the domain of the closure of $\pi_{k}^{+}\left(B_{c}\right)$ equals the domain of the self-adjoint extension of $\pi_{k}^{+}\left(H_{c}\right)$. In particular, $v_{x}^{+}$is in the domain of the closure of $\pi_{k}^{+}\left(B_{c}\right)$, and similarly for $\pi_{k}^{+}\left(C_{c}\right)$ and for the other representations. From the commutation relations we get $\pi_{k}^{+}\left(B_{c}\right) v_{x}^{+}=\alpha v_{x+1}^{+}$. By proposition 3.1

$$
\alpha\left\langle v_{x+1}^{+}, e_{0}\right\rangle=\left\langle\pi_{k}^{+}\left(B_{c}\right) v_{x}^{+}, e_{0}\right\rangle=-\left\langle v_{x}^{+}, \pi_{k}^{+}\left(C_{c}\right) e_{0}\right\rangle=-\sqrt{w(x ; 2 k, c)}\left(\frac{2 k \sqrt{c}}{1-c}-\frac{c \sqrt{2 k}}{1-c} \hat{M}_{1}(x ; 2 k, c)\right) .
$$

From the recurrence relation for the Meixner polynomials, we find $\hat{M}_{1}(x ; 2 k, c)=\sqrt{2 k c}-\frac{(1-c) x}{\sqrt{2 k c}}$ and for the weight function we have

$$
\frac{w(x ; 2 k, c)}{w(x+1 ; 2 k, c)}=\frac{x+1}{(2 k+x) c} .
$$

So we find

$$
\pi^{+}\left(B_{c}\right) v_{x}^{+}=-\sqrt{(x+1)(2 k+x)} v_{x+1}^{+} .
$$

In the same way we can find the actions of $B_{c}$ and $C_{c}$ in the discrete series. For the principal unitary and the complementary series, we use the same method, but since there is no highest/lowest weight vector in the representation space, we also need the following contiguous relations

$$
\begin{aligned}
c(1-c)(1-z){ }_{2} F_{1}\left(\begin{array}{c}
a, b \\
c-1
\end{array} ; z\right)= & (c-a)(c-b) z_{2} F_{1}\left(\begin{array}{c}
a, b \\
c+1
\end{array} ; z\right) \\
& +c(c-1-(2 c-a-b-1) z){ }_{2} F_{1}\left(\begin{array}{c}
a, b \\
c
\end{array} ; z\right),
\end{aligned}
$$

and

$$
{ }_{2} F_{1}\left(\begin{array}{c}
a, b \\
c
\end{array} ; z\right)={ }_{2} F_{1}\left(\begin{array}{c}
a, b \\
c-1
\end{array} ; z\right)-\frac{a b z}{c(c-1)}{ }_{2} F_{1}\left(\begin{array}{c}
a+1, b+1 \\
c+1
\end{array} ; z\right)
$$


For the first relation, see [3, $\S 2.8$, eq. 30]; the second relation follows directly from

$$
(c-1)_{n}=\frac{c-1}{c-1+n}(c)_{n} .
$$

Then we have

$$
\begin{gathered}
\pi_{k}^{+}\left(H_{c}\right) v_{x}^{+}=2(k+x) v_{x}^{+}, \\
\pi_{k}^{+}\left(B_{c}\right) v_{x}^{+}=-\sqrt{(x+1)(2 k+x)} v_{x+1}^{+}, \\
\pi_{k}^{+}\left(C_{c}\right) v_{x}^{+}=\sqrt{x(2 k+x-1)} v_{x-1}^{+}, \\
\pi_{k}^{-}\left(H_{c}\right) v_{x}^{-}=2(k+x) v_{x}^{-}, \\
\pi_{k}^{-}\left(B_{c}\right) v_{x}^{-}=\sqrt{x(2 k+x-1)} v_{x-1}^{-}, \\
\pi_{k}^{-}\left(C_{c}\right) v_{x}^{-}=-\sqrt{(x+1)(2 k+x)} v_{x+1}^{-}, \\
\pi^{\lambda, \varepsilon}\left(H_{c}\right) w_{x}^{\lambda, \varepsilon}=2(\varepsilon+x) w_{x}^{\lambda, \varepsilon}, \\
\pi^{\lambda, \varepsilon}\left(B_{c}\right) w_{x}^{\lambda, \varepsilon}=\sqrt{(x+\varepsilon-\lambda)(x+\varepsilon+\lambda+1)} w_{x+1}^{\lambda, \varepsilon}, \\
\pi^{\lambda, \varepsilon}\left(C_{c}\right) w_{x}^{\lambda, \varepsilon}=-\sqrt{(x+\varepsilon+\lambda)(x+\varepsilon-\lambda-1)} w_{x-1}^{\lambda, \varepsilon} .
\end{gathered}
$$

Comparing these actions to the actions of $H, B$ and $C$ on the basisvectors $e_{n}$ for the representations in (2.2), (2.3), (2.4) and (2.5), we see that the actions are equivalent, where $x$ plays the role of $n$. Observe that, to get exactly the same actions in the discrete series representations, we have to let $H_{c}, B_{c}$ and $C_{c}$ act on $(-1)^{x} v_{x}$.

Proposition 3.4. For $x_{1}, x_{2} \in \mathbb{Z}_{\geq 0}, v_{x_{1}}^{+} \otimes v_{x_{2}}^{-}$is an eigenvector of $\pi_{k_{1}}^{+} \otimes \pi_{k_{2}}^{-}\left(\Delta\left(X_{c}\right)\right)$ for the eigenvalue $(c-1)\left(x_{1}-x_{2}+k_{1}-k_{2}\right) / \sqrt{c}$. And

$$
\begin{aligned}
v_{x_{1}}^{+} \otimes v_{x_{2}}^{-} & =\sum_{n_{1}=0}^{\infty} \sum_{n_{2}=0}^{\infty} \hat{M}_{n_{1}}\left(x_{1} ; 2 k_{1}, c\right) \hat{M}_{n_{2}}\left(x_{2} ; 2 k_{2}, c\right) \sqrt{w\left(x_{1} ; 2 k_{1}, c\right) w\left(x_{2} ; 2 k_{2}, c\right)} e_{n_{1}} \otimes e_{n_{2}} \\
& =(-1)^{x_{1}} \int_{\mathbb{R}} \hat{S}_{x}\left(y ; x_{1}-x_{2}\right) v_{x_{1}-x_{2}-L} d \mu\left(y ; x_{1}-x_{2}\right),
\end{aligned}
$$

where $x=\min \left\{x_{1}, x_{2}\right\}$ and $v_{x_{1}-x_{2}-L}$ is the eigenvector for $X_{c}$ in the principal, complementary or negative discrete series representation, depending on the value of $y$ in the same way as in theorem 2.9.

The second expression of (3.9) states that the continuous dual Hahn polynomials have an interpretation as Clebsch-Gordan coefficients for the basis of eigenvectors of $X_{c}$.

Proof. That $v_{x_{1}}^{+} \otimes v_{x_{2}}^{-}$is an eigenvector of $\pi_{k_{1}}^{+} \otimes \pi_{k_{2}}^{-}\left(\Delta\left(H_{c}\right)\right)$ for the eigenvalue $2\left(x_{1}-x_{2}+k_{1}-k_{2}\right)$ follows from proposition 3.1. This also gives the first expression for $v_{x_{1}}^{+} \otimes v_{x_{2}}^{-}$. We find the second expression using (2.9) in theorem 2.2 and the observations above.

Remark 3.5. We indicate an alternative proof of proposition 3.4 assuming, for simplicity, $k_{1}-k_{2} \geq$ $-\frac{1}{2}$ and $k_{1}+k_{1} \leq \frac{1}{2}$. From propositions 3.1, 3.2 and theorem 2.2 we get that

$$
v_{x_{1}}^{+} \otimes v_{x_{2}}^{-}=\int_{0}^{\infty} F\left(y ; x_{1}, x_{2}\right) v_{x_{1}-x_{2}-L}^{\sqrt{y, \varepsilon}} d \mu\left(y ; x_{1}-x_{2}\right)
$$


for some function $F$ to be determined. Taking inner products with $e_{0} \otimes e_{0}=\int_{0}^{\infty} e_{-L} d \mu(y ; 0)$ gives

$$
\begin{aligned}
\sqrt{w\left(x_{1} ; 2 k_{1}, c\right) w\left(x_{2} ; 2 k_{2}, c\right)}=\int_{0}^{\infty} & F\left(y ; x_{1}, x_{2}\right) \hat{m}_{-L}\left(x_{1}-x_{2}-L ;-\frac{1}{2}+i \sqrt{y}, \varepsilon, c\right) \\
& \times \sqrt{\widetilde{w}\left(x_{1}-x_{2}-L ;-\frac{1}{2}+i \sqrt{y}, \varepsilon, c\right)} d \mu\left(y ; x_{1}-x_{2}\right)
\end{aligned}
$$

and since $\hat{m}_{-L}\left(x_{1}-x_{2}-L ;-\frac{1}{2}+i \sqrt{y}, \varepsilon, c\right)$ can be expressed in terms of $\varphi_{2 \sqrt{y}}^{\left(x_{2}-x_{1}, x_{1}-x_{2}-2 L+2 \varepsilon\right)}$, see (3.8), $F$ is the inverse Jacobi transform [12] of elementary functions. This Jacobi transform can be calculated explicitly using a limit case of Koornwinder's formula [12, eq. (9.4)] stating that Jacobi polynomials are mapped onto the Wilson polynomials. Using simple expansions and Saalschütz's and Kummer's summation formulas the explicit form for $F$ in terms of a terminating ${ }_{3} F_{2}$-series can be derived.

3.3. Bilinear generating functions for Meixner polynomials. In this subsection we use the result of proposition 3.4 to find a bilinear generating functions for Meixner polynomials.

We write the second expression of (3.9) as

$$
\begin{aligned}
&(-1)^{x_{1}} \int_{\mathbb{R}} \hat{S}_{x}\left(y ; x_{1}-x_{2}\right) v_{x_{1}-x_{2}-L} d \mu\left(y ; x_{1}-x_{2}\right)= \\
& \sum_{r=-\infty}^{\infty} \int_{\mathbb{R}} \hat{S}_{x}\left(y ; x_{1}-x_{2}\right) \hat{m}_{r}\left(x_{1}-x_{2}-L ;-\frac{1}{2}+i \sqrt{y}, \varepsilon, c\right) \\
& \quad \times \sqrt{\widetilde{w}\left(x_{1}-x_{2}-L ;-\frac{1}{2}+i \sqrt{y}, \varepsilon, c\right)} e_{r} d \mu\left(y ; x_{1}-x_{2}\right) .
\end{aligned}
$$

To show that this also covers the case $k_{1}-k_{2}<-\frac{1}{2}$, where we should have a finite sum of eigenvectors $v^{-}$, observe that for $y=-\left(k_{1}-k_{2}+\frac{1}{2}+j\right)=-\left(\varepsilon-L+\frac{1}{2}+j\right), 0 \leq j \leq K$, the ${ }_{2} F_{1}$-series in the Meixner function terminates. Reversing the sum

$$
{ }_{2} F_{1}\left(\begin{array}{c}
-n, b \\
c
\end{array} ; x\right)=(-x)^{n} \frac{(b)_{n}}{(c)_{n}}{ }_{2} F_{1}\left(\begin{array}{c}
-n,-c-n+1 \\
-b-n+1
\end{array} ; \frac{1}{x}\right) .
$$

then gives

$$
\begin{gathered}
\hat{m}_{n_{1}-n_{2}-L}\left(x_{1}-x_{2}-L ;-\frac{1}{2}+i \sqrt{y}, k_{1}-k_{2}+L, c\right) \sqrt{\widetilde{w}\left(x_{1}-x_{2}-L ;-\frac{1}{2}+i \sqrt{y}, k_{1}-k_{2}+L, c\right)} \\
=\hat{M}_{j-n_{1}+n_{2}}\left(j-x_{1}+x_{2} ; 2 k_{2}-2 k_{1}-2 j, c\right) \sqrt{w\left(j-x_{1}+x_{2} ; 2 k_{2}-2 k_{1}-2 j, c\right)} .
\end{gathered}
$$

So indeed we find a finite sum of eigenvectors $v_{j-x_{1}+x_{2}}^{-}$.

Now use (2.10) to find

$$
\begin{aligned}
& \left\langle v_{x_{1}}^{+} \otimes v_{x_{2}}^{-}, e_{n_{1}} \otimes e_{n_{2}}\right\rangle= \\
& (-1)^{x_{1}+n_{2}} \int_{\mathbb{R}} \hat{S}_{n}\left(y ; n_{1}-n_{2}\right) \hat{S}_{x}\left(y ; x_{1}-x_{2}\right) \hat{m}_{n_{1}-n_{2}-L}\left(x_{1}-x_{2}-L ;-\frac{1}{2}+i \sqrt{y}, \varepsilon, c\right) \\
& \quad \times \sqrt{\widetilde{w}\left(x_{1}-x_{2}-L ;-\frac{1}{2}+i \sqrt{y}, \varepsilon, c\right)} d \nu\left(y ; n_{1}-n_{2}, x_{1}-x_{2}\right),
\end{aligned}
$$

where $d \nu(\cdot ; n, x)$ is the restriction to the diagonal of the product measure obtained from the measures $d \mu(\cdot ; n)$ and $d \mu(\cdot ; x)$. From the first expression of (3.9) we find

$$
\left\langle v_{x_{1}}^{+} \otimes v_{x_{2}}^{-}, e_{n_{1}} \otimes e_{n_{2}}\right\rangle=\hat{M}_{n_{1}}\left(x_{1} ; 2 k_{1}, c\right) \hat{M}_{n_{2}}\left(x_{2} ; 2 k_{2}, c\right) \sqrt{w\left(x_{1} ; 2 k_{1}, c\right) w\left(x_{2} ; 2 k_{2}, c\right)},
$$


so we have found the following formula

$$
\begin{aligned}
(-1)^{n_{2}+x_{1}} \int_{\mathbb{R}} & \hat{S}_{n}\left(y ; n_{1}-n_{2}\right) \hat{S}_{x}\left(y ; x_{1}-x_{2}\right) \hat{m}_{n_{1}-n_{2}-L}\left(x_{1}-x_{2}-L ;-\frac{1}{2}+i \sqrt{y}, \varepsilon, c\right) \\
& \times \sqrt{\widetilde{w}\left(x_{1}-x_{2}-L ;-\frac{1}{2}+i \sqrt{y}, \varepsilon, c\right)} d \nu\left(y ; n_{1}-n_{2}, x_{1}-x_{2}\right) \\
= & \hat{M}_{n_{1}}\left(x_{1} ; 2 k_{1}, c\right) \hat{M}_{n_{2}}\left(x_{2} ; 2 k_{2}, c\right) \sqrt{w\left(x_{1} ; 2 k_{1}, c\right) w\left(x_{2} ; 2 k_{2}, c\right)} .
\end{aligned}
$$

This can be interpreted as a connection formula between two orthogonal bases in $L^{2}\left(\mathbb{Z}_{\geq 0}^{2}\right)$.

Theorem 3.6. The continuous dual Hahn and the Meixner polynomials, in the notations (2.6) and (3.1), satisfy, for $k_{2} \geq k_{1}$,

$$
\begin{aligned}
& S_{x_{2}}\left(y ; k_{2}-k_{1}+\frac{1}{2}, k_{1}+k_{2}-\frac{1}{2}, k_{1}-k_{2}+x_{1}-x_{2}+\frac{1}{2}\right) \\
& \quad \times \frac{1}{\Gamma\left(p+x_{2}-x_{1}+1\right)}{ }_{2} F_{1}\left(\begin{array}{c}
p+k_{1}-k_{2}+\frac{1}{2}+i \sqrt{y}, p+k_{1}-k_{2}+\frac{1}{2}-i \sqrt{y} ; \frac{c}{p+x_{2}-x_{1}+1} \\
p-1
\end{array}\right) \\
& \quad=\sum_{n=0}^{\infty} C_{n} S_{n}\left(y ; k_{2}-k_{1}+\frac{1}{2}, k_{1}+k_{2}-\frac{1}{2}, k_{1}-k_{2}+p+\frac{1}{2}\right) M_{n+p}\left(x_{1} ; 2 k_{1}, c\right) M_{n}\left(x_{2} ; 2 k_{2}, c\right),
\end{aligned}
$$

where

$$
C_{n}=(-1)^{x_{1}+x_{2}} \frac{c^{n+x_{1}}(1-c)^{2 k_{1}+p}\left(2 k_{1}\right)_{x_{1}}\left(2 k_{2}\right)_{x_{2}}}{\Gamma(n+1) \Gamma(n+p+1)} .
$$

Proof. First we concentrate on the case $x_{1} \geq x_{2}$. Using the orthonormality of $\hat{S}_{n}$ in (3.10) and writing the polynomials in the usual normalization, we find

$$
\begin{aligned}
& S_{x_{2}}\left(y ; k_{2}-k_{1}+\frac{1}{2}, k_{1}+k_{2}-\frac{1}{2}, k_{1}-k_{2}+x_{1}-x_{2}+\frac{1}{2}\right) \\
& \times \frac{1}{\Gamma\left(p+x_{2}-x_{1}+1\right)}{ }_{2} F_{1}\left(\begin{array}{c}
\left.p+k_{1}-k_{2}+\frac{1}{2}+i \sqrt{y}, p+k_{1}-k_{2}+\frac{1}{2}-i \sqrt{y} ; \frac{c}{c-1}\right) \\
p+x_{2}-x_{1}+1
\end{array}\right) \\
& =\left\{\begin{array}{c}
\sum_{n=0}^{\infty} C_{n}^{-} S_{n}\left(y ; k_{1}-k_{2}+\frac{1}{2}, k_{1}+k_{2}-\frac{1}{2}, k_{2}-k_{1}-p+\frac{1}{2}\right) \\
\times M_{n}\left(x_{1} ; 2 k_{1}, c\right) M_{n-p}\left(x_{2} ; 2 k_{2}, c\right), \quad p \leq 0, \\
\sum_{n=0}^{\infty} C_{n}^{+} S_{n}\left(y ; k_{2}-k_{1}+\frac{1}{2}, k_{1}+k_{2}-\frac{1}{2}, k_{1}-k_{2}+p+\frac{1}{2}\right) \\
\times M_{n+p}\left(x_{1} ; 2 k_{1}, c\right) M_{n}\left(x_{2} ; 2 k_{2}, c\right), \quad p \geq 0,
\end{array}\right.
\end{aligned}
$$

where

$$
\begin{aligned}
C_{n}^{-}=(-1)^{x_{1}+x_{2}+p} & \frac{c^{n-p+x_{1}}(1-c)^{2 k_{1}+p}\left(2 k_{1}\right)_{x_{1}}\left(2 k_{2}\right)_{x_{2}}}{\Gamma(n+1) \Gamma(n-p+1)} \\
& \times\left|\frac{\Gamma\left(k_{1}-k_{2}+\frac{1}{2}+i \sqrt{y}\right) \Gamma\left(k_{2}-k_{1}-p+\frac{1}{2}+i \sqrt{y}\right)}{\Gamma\left(k_{1}-k_{2}+p+\frac{1}{2}+i \sqrt{y}\right) \Gamma\left(k_{2}-k_{1}+\frac{1}{2}+i \sqrt{y}\right)}\right|
\end{aligned}
$$

and

$$
C_{n}^{+}=(-1)^{x_{1}+x_{2}} \frac{c^{n+x_{1}}(1-c)^{2 k_{1}+p}\left(2 k_{1}\right)_{x_{1}}\left(2 k_{2}\right)_{x_{2}}}{\Gamma(n+1) \Gamma(n+p+1)} .
$$

Now consider the expression on the right hand side of (3.12) for $p \leq 0$. This expression is also well defined for $p \geq 0$, since then the factor $(\Gamma(n-p+1))^{-1}$, which equals zero for $n<p$, makes 
the summation start at $n=p$ instead of $n=0$. Using (2.6) and writing out the ${ }_{3} F_{2}$-series in the continuous dual Hahn polynomial as a sum, we get

$$
\begin{aligned}
S_{n}\left(y ; k_{1}-k_{2}+\frac{1}{2}, k_{1}+k_{2}-\frac{1}{2}, k_{2}-k_{1}-p+\frac{1}{2}\right) \\
=\frac{\Gamma\left(2 k_{1}+n\right) \Gamma(n-p+1)}{\Gamma\left(2 k_{1}\right)} \sum_{j=p}^{n} \frac{(-n)_{j}\left(k_{1}-k_{2}+\frac{1}{2}+i \sqrt{y}\right)_{j}\left(k_{1}-k_{2}+\frac{1}{2}-i \sqrt{y}\right)_{j}}{\left(2 k_{1}\right)_{j} \Gamma(1-p+j)(1)_{j}} \\
=(-1)^{p}\left|\frac{\Gamma\left(k_{1}-k_{2}+p+\frac{1}{2}+i \sqrt{y}\right)}{\Gamma\left(k_{1}-k_{2}+\frac{1}{2}+i \sqrt{y}\right)}\right|^{2} \frac{\Gamma\left(2 k_{1}+n\right) \Gamma(n+1)}{\Gamma\left(2 k_{1}+p\right) \Gamma(p+1)} \\
\quad \times \sum_{l=0}^{n-p} \frac{(p-n)_{l}\left(k_{1}-k_{2}+p+\frac{1}{2}+i \sqrt{y}\right)_{l}\left(k_{1}-k_{2}+p+\frac{1}{2}-i \sqrt{y}\right)_{l}}{\left(2 k_{1}+p\right)_{l}(p+1)_{l} l !} \\
=(-1)^{p}\left|\frac{\Gamma\left(k_{1}-k_{2}+p+\frac{1}{2}+i \sqrt{y}\right)}{\Gamma\left(k_{1}-k_{2}+\frac{1}{2}+i \sqrt{y}\right)}\right|^{2} S_{n-p}\left(y ; k_{2}-k_{1}+\frac{1}{2}, k_{1}+k_{2}-\frac{1}{2}, k_{1}-k_{2}+p-\frac{1}{2}\right) .
\end{aligned}
$$

Now we shift the index $n$ in the expression for $p \leq 0$ of (3.12) to $n+p$, and we use the reflection formula for the $\Gamma$-function, to see that the expressions for $p \leq 0$ and $p \geq 0$ in (3.12) are the same.

For $x_{1} \leq x_{2}$ we get from (3.10) a formula similar to (3.12), with $S_{x_{1}}\left(y ; k_{1}-k_{2}+\frac{1}{2}, k_{1}+k_{2}-\right.$ $\left.\frac{1}{2}, k_{2}-k_{1}+x_{2}-x_{1}+\frac{1}{2}\right)$ as continuous dual Hahn polynomial on the left hand side and different $\Gamma$-factors in the constants $C_{n}^{+}$and $C_{n}^{-}$on the right hand side. We use (3.13) again to see that both expressions on the right hand side are the same. Then we use (3.13) again with $p=x_{1}-x_{2}$ and the reflection formula for the $\Gamma$-function, to obtain exactly the same formula as for $x_{1} \geq x_{2}$.

Remark 3.7. Several special cases of theorem 3.6 are of special interest, or reduce to well-known results.

(i) For $x_{2}=0$ the continuous dual Hahn polynomial on the left hand side of (3.11) and $M_{n}\left(x_{2} ; 2 k_{2}, c\right)$ reduce to 1 . This gives the expansion of a Jacobi function, as function of $y$, in terms of continuous dual Hahn polynomials with Meixner polynomials as coefficients.

(ii) For $x_{1}=x_{2}=0$ in (3.11) we find a special case of the generating function for continuous dual Hahn polynomials

$$
(1-t)^{-\gamma}{ }_{3} F_{2}\left(\begin{array}{c}
\gamma, a+i x, a-i x \\
a+b, a+c
\end{array} ; \frac{t}{t-1}\right)=\sum_{n=0}^{\infty} \frac{(\gamma)_{n} S_{n}\left(x^{2} ; a, b, c\right)}{(a+b)_{n}(a+c)_{n} n !} t^{n}, \quad \gamma \text { arbitrary }
$$

(iii) For $p=0, k_{1}=k_{2}$ and values of $y$ such that the ${ }_{3} F_{2}$-series in the continuous dual Hahn polynomials becomes summable by Saalschütz theorem [3, §2.1.5, eq. 30]

$$
{ }_{3} F_{2}\left(\begin{array}{c}
-n, a, b \\
c, 1+a+b-c-n
\end{array} ; 1\right)=\frac{(c-a)_{n}(c-b)_{n}}{(c)_{n}(c-a-b)_{n}},
$$

we find the dual orthogonality relations (3.3) for the Meixner polynomials, which are equivalent to the orthogonality relations of the Meixner polynomials.

(iv) An expression somewhat similar to (3.11), but simpler in structure, for the Al-Salam and Chihara polynomials can be found in [6, Thm. 4.3]

Remark 3.8. The element $X_{c}$, which we considered in section 3.1, is a special case of the element $X_{a}=-2 a H+B-C \in \mathfrak{s u}(1,1)$, namely the case for $|a|>1$. For $|a|<1$ and $|a|=1$ this element $X_{a}$ in the discrete series representations has a continuous spectrum $(-\infty, \infty)$ and $[0, \infty)$, which corresponds to Meixner-Pollaczek and Laguerre polynomials respectively (see Koelink and Van der Jeugt [10, §3.1] and Van der Jeugt [16, §2]). But in the principal and the complementary 
series, the corresponding functions give much more difficulties than the Meixner functions we used in this paper. For $|a|<1$ the spectral measure is calculated in [9, ex. 4.4.11] and it turns out that the spectral projection is on a two-dimensional space of generalized eigenvectors. So we find orthogonality relations involving two linearly independent "Meixner-Pollaczek"-functions, instead of just one function as for $|a|>1$. This makes it much harder to compute the Clebsch-Gordan coefficients for the bases of (generalized) eigenvectors of $X_{a}$ in this way. However Hjalmar Rosengren pointed us that the calculations of the Clebsch-Gordan coefficients might be possible from proving the analogue of (3.11) by brute force. In particular, Rosengren can prove (3.11) by direct methods.

\section{HolOMORPHIC AND ANTI-HOLOMORPHIC FUNCTIONS}

In this section we choose explicit realizations of $\mathfrak{s u}(1,1)$ and the basisvectors $e_{n}$. Using these in (3.9) we find a bilinear generating function for the Meixner functions.

Consider the Hilbert space of holomorphic functions on the unit disk $D$, with finite norm with respect to the inner product

$$
\langle f, g\rangle=\sum_{n=0}^{\infty} \frac{n !}{(2 k)_{n}} f_{n} \overline{g_{n}}, \quad f(z)=\sum_{n=0}^{\infty} f_{n} z^{n} .
$$

The standard orthonormal basisvectors in this space are

$$
e_{n}=e_{n}^{k}(z)=\sqrt{\frac{(2 k)_{n}}{n !}} z^{n} .
$$

The realization of the positive discrete series representation is

$$
\begin{aligned}
& \pi_{k}^{+}(H)=2 z \frac{d}{d z}+2 k \\
& \pi_{k}^{+}(B)=z^{2} \frac{d}{d z}+2 k z, \\
& \pi_{k}^{+}(C)=-\frac{d}{d z}
\end{aligned}
$$

An eigenvector for $\pi_{k}^{+}\left(X_{c}\right)$ for the eigenvalue $(c-1)(x+k) / \sqrt{c}$ is

$$
v_{x}^{+}=\sum_{n=0}^{\infty} \hat{M}_{n}(x ; 2 k, c) e_{n}^{+}, \quad x \in \mathbb{Z}_{+} .
$$

Using the orthonormal basisvectors above, this eigenvector becomes a generating function for the orthonormal Meixner polynomials (see [2, p.349] or [8])

$$
v_{x}^{+}(z)=\sum_{n=0}^{\infty} \sqrt{\frac{(2 k)_{n}}{n !}} \hat{M}_{n}(x ; 2 k, c) z^{n}=\left(1-\frac{z}{\sqrt{c}}\right)^{x}(1-\sqrt{c} z)^{-x-2 k},
$$

where $x \in \mathbb{Z}_{\geq 0}$. This is a holomorphic function for $|z|<1 / \sqrt{c}$. The right hand side can also be found by solving the first order differential equation

$$
\pi_{k}^{+}\left(X_{c}\right) y(z)=\frac{(c-1)(x+k)}{\sqrt{c}} y(z), \quad y(0)=1 .
$$

Consider the Hilbert space of anti-holomorphic functions on the unit disk $D$, with the same inner product as above, now using $f(z)=\sum_{n=0}^{\infty} f_{n} \bar{z}^{n}$. The standard orthonormal basisvectors in this space are

$$
e_{n}=e_{n}^{k}(\bar{z})=\sqrt{\frac{(2 k)_{n}}{n !}} \bar{z}^{n}
$$


The realization of the negative discrete series representation is

$$
\begin{aligned}
& \pi_{k}^{+}(H)=-2 \bar{z} \frac{d}{d \bar{z}}-2 k \\
& \pi_{k}^{+}(B)=-\frac{d}{d \bar{z}} \\
& \pi_{k}^{+}(C)=\bar{z}^{2} \frac{d}{d \bar{z}}+2 k \bar{z} .
\end{aligned}
$$

An eigenvector for $\pi_{k}^{-}\left(X_{c}\right)$ for the eigenvalue $(1-c)(x+k) / \sqrt{c}$ is

$$
v_{x}^{-}=\sum_{n=0}^{\infty} \hat{M}_{n}(x ; 2 k, c) e_{n}^{-}=\left(1-\frac{\bar{z}}{\sqrt{c}}\right)^{x}(1-\sqrt{c} \bar{z})^{-x-2 k},
$$

where $x \in \mathbb{Z}_{+}$. This is an anti-holomorphic function for $|z|<1 / \sqrt{c}$.

Next we use (2.10) to realize $f \otimes e_{r-L}$ in the tensor product representation as functions of $z$ and $\bar{w}$. To make calculations easier we will assume $k_{1}-k_{2} \geq-\frac{1}{2}$ and $k_{1}+k_{2} \geq \frac{1}{2}$, so the orthogonality measure for the continuous dual Hahn polynomials has only an absolutely continuous part. The result remains valid in the general case.

Proposition 4.1. In the explicit realizations (4.1) and (4.3), we have for $|z \bar{w}|<1$

$$
\begin{aligned}
& \left(f \otimes e_{r-L}\right)(z, \bar{w})= \\
& \frac{z^{r}(1-z \bar{w})^{-2 k_{2}}}{\sqrt{2 \pi \Gamma\left(2 k_{1}\right) \Gamma\left(2 k_{2}\right)}} \int_{0}^{\infty} f\left(\rho^{2}\right)\left|\frac{\Gamma\left(k_{1}-k_{2}+r+\frac{1}{2}+i \rho\right) \Gamma\left(k_{1}+k_{2}-\frac{1}{2}+i \rho\right) \Gamma\left(k_{2}-k_{1}+\frac{1}{2}+i \rho\right)}{\Gamma(2 i \rho)}\right| \\
& \quad \times \frac{1}{\Gamma(1+r)}{ }_{2} F_{1}\left(\begin{array}{c}
\left.k_{2}-k_{1}+\frac{1}{2}+i \rho, k_{2}-k_{1}+\frac{1}{2}-i \rho ; \frac{z \bar{w}}{z \bar{w}-1}\right) d \rho . \\
1+r
\end{array}\right.
\end{aligned}
$$

Proof. Substitute (4.1) and (4.3) in (2.10), interchange summation and integration, use the generating function for continuous dual Hahn polynomials [8], [2, p. 349]

$$
\sum_{n=0}^{\infty} \frac{S_{n}\left(x^{2} ; a, b, c\right)}{n !(a+c)_{n}} t^{n}=(1-t)^{-b+i x}{ }_{2} F_{1}\left(\begin{array}{c}
a+i x, c+i x \\
a+c
\end{array} ; t\right), \quad|t|<1,
$$

and the transformation [3, $\S 2.1 .4$, eq. 22]

$$
{ }_{2} F_{1}\left(\begin{array}{c}
a, b \\
c
\end{array} ; t\right)=(1-t)^{-a}{ }_{2} F_{1}\left(\begin{array}{c}
a, c-b \\
c
\end{array} \frac{t}{t-1}\right),
$$

then we find (4.5) for $r \geq 0$. Following the same procedure gives for $r \leq 0$

$$
\begin{aligned}
\left(f \otimes e_{r-L}\right)(z, \bar{w}) & =\frac{(-1)^{r} \bar{w}^{-r}(1-z \bar{w})^{-2 k_{1}}}{\sqrt{2 \pi \Gamma\left(2 k_{1}\right) \Gamma\left(2 k_{2}\right)}} \\
& \times \int_{0}^{\infty} f\left(\rho^{2}\right)\left|\frac{\Gamma\left(k_{1}-k_{2}+\frac{1}{2}+i \rho\right) \Gamma\left(k_{1}+k_{2}-\frac{1}{2}+i \rho\right) \Gamma\left(k_{2}-k_{1}-r+\frac{1}{2}+i \rho\right) \mid}{\Gamma(2 i \rho)}\right| \\
& \times \frac{1}{\Gamma(1-r)}{ }_{2} F_{1}\left(\begin{array}{c}
k_{1}-k_{2}+\frac{1}{2}+i \rho, k_{1}-k_{2}+\frac{1}{2}-i \rho \\
1-r
\end{array} ; \frac{z \bar{w}}{z \bar{w}-1}\right) d \rho .
\end{aligned}
$$

Use [3, §2.1.4, eq. 23]

$$
{ }_{2} F_{1}\left(\begin{array}{c}
a, b \\
c
\end{array} ; t\right)=(1-t)^{c-a-b}{ }_{2} F_{1}\left(\begin{array}{c}
c-a, c-b \\
c
\end{array} ; t\right)
$$


and for $r \in \mathbb{Z}$

$$
\begin{aligned}
\frac{1}{\Gamma(1-r)}{ }_{2} F_{1}\left(\begin{array}{c}
a, b \\
1-r
\end{array} ; t\right) & =\sum_{k=r}^{\infty} \frac{(a)_{k}(b)_{k}}{\Gamma(1-r+k) k !} t^{k}= \\
\frac{(a)_{r}(b)_{r} t^{r}}{r !} \sum_{p=0}^{\infty} \frac{(a+r)_{p}(b+r)_{p}}{(r+1)_{p} p !} t^{p} & =\frac{\Gamma(a+r) \Gamma(b+r)}{\Gamma(a) \Gamma(b)} \frac{t^{r}}{\Gamma(1+r)}{ }_{2} F_{1}\left(\begin{array}{c}
a+r, b+r \\
r+1
\end{array} ; t\right),
\end{aligned}
$$

and the reflection formula for the $\Gamma$-function, to see that the expressions for $r \leq 0$ and $r \geq 0$ are the same.

Remark 4.2. Note that the ${ }_{2} F_{1}$-series inside the integral is a Jacobi function. So we can consider $f \otimes e_{n}$ in this realization as the inverse Jacobi transform of the function $f$ times $\Gamma$-functions.

Next we consider the eigenvectors in this realization. Using proposition 4.1 and interchanging summation and integration, we find

$$
\begin{aligned}
\left(f \otimes v_{x-L}\right)(z, \bar{w}) & =\frac{(1-z \bar{w})^{-2 k_{2}}}{\sqrt{2 \pi \Gamma\left(2 k_{1}\right) \Gamma\left(2 k_{2}\right)}} \int_{0}^{\infty} f\left(\rho^{2}\right)\left|\frac{\Gamma\left(k_{2}-k_{1}+\frac{1}{2}+i \rho\right) \Gamma\left(k_{1}+k_{2}-\frac{1}{2}+i \rho\right)}{\Gamma(2 i \rho)}\right| \\
& \times \sqrt{\widetilde{w}(x-L ; \rho, \varepsilon, c)} \sum_{n=-\infty}^{\infty} \hat{m}_{n-L}\left(x-L ;-\frac{1}{2}+i \rho, \varepsilon, c\right) \frac{\left|\Gamma\left(n+k_{1}-k_{2}+\frac{1}{2}+i \rho\right)\right|}{\Gamma(1+n)} \\
& \times{ }_{2} F_{1}\left(\begin{array}{c}
k_{2}-k_{1}+\frac{1}{2}+i \rho, k_{2}-k_{1}+\frac{1}{2}-i \rho \\
1+n
\end{array} ; \frac{z \bar{w}}{z \bar{w}-1}\right) z^{n} d \rho
\end{aligned}
$$

Note that the ${ }_{2} F_{1}$-series has the same form, after applying transformation (4.6), as the ${ }_{2} F_{1}$-series in definition of the Meixner function. So we may consider the inner sum as a non-symmetric Poisson kernel for the Meixner functions. To find conditions for the convergence of the inner sum, we look at the asymptotic behaviour of the Meixner function. The asymptotic behaviour has been determined in $[9, \S 4.4]$ and $[13, \S 3]$. We have

$$
\hat{m}_{n}(x ; \lambda, \varepsilon, c)=C_{1} n^{x+\varepsilon-\frac{1}{2}} c^{n / 2}\left(1+\mathcal{O}\left(\frac{1}{n}\right)\right), \quad n \rightarrow \infty,
$$

where $C_{1}$ is a non-zero constant independent of $n$. The asymptotics as $n \rightarrow-\infty$ follows from the connection formula [13, eq.3.8], [9, eq.4.4.7] and observing that one term vanishes for $x \in \mathbb{Z}$. This gives

$$
m_{-n}(x ; \lambda, \varepsilon, c)=C_{2} n^{-x-\varepsilon-\frac{1}{2}} c^{n / 2}\left(1+\mathcal{O}\left(\frac{1}{n}\right)\right), \quad n \rightarrow \infty,
$$

where $C_{2}$ is a non-zero constant independent of $n$. Now we find that the inner sum in (4.8) converges for $|z|<1 / \sqrt{c}$ and $|w|<1 / \sqrt{c}$. So for these conditions we may indeed interchange summation and integration for sufficiently smooth $f$, e.g. $f \in \mathcal{S}$.

Using (4.2) and (4.4), we find an expression for $v_{p}^{+} \otimes v_{p-x}^{-}, x \leq 0$, in this realization

$$
\begin{aligned}
& \left(v_{p}^{+} \otimes v_{p-x}^{-}\right)(z, \bar{w})=v_{p}^{+}(z) \otimes v_{p-x}^{-}(\bar{w})= \\
& (1-z \sqrt{c})^{-2 k_{1}}(1-\bar{w} \sqrt{c})^{-2 k_{2}}(1-c)^{k_{1}+k_{2}} \sqrt{\frac{\left(2 k_{1}\right)_{p}}{p !}}\left(\frac{\sqrt{c}-z}{1-z \sqrt{c}}\right)^{p} \sqrt{\frac{\left(2 k_{2}\right)_{p-x}}{(p-x) !}}\left(\frac{\sqrt{c}-\bar{w}}{1-\bar{w} \sqrt{c}}\right)^{p-x} .
\end{aligned}
$$

Observe that this has exactly the same structure as the realization for $e_{n} \otimes e_{n-r}$. In the same way we can give an expression for $v_{p+x}^{+} \otimes v_{p}^{-}, x \geq 0$. Now we use the analogue of (2.10) for the 
eigenvectors and the substitutions

$$
r \mapsto x, \quad z \mapsto \frac{\sqrt{c}-z}{1-z \sqrt{c}}, \quad \bar{w} \mapsto \frac{\sqrt{c}-\bar{w}}{1-\bar{w} \sqrt{c}},
$$

to find another expression for $f \otimes v_{x-L}$ in this realization,

$$
\begin{aligned}
& \left(f \otimes v_{x-L}\right)(z, \bar{w})=(-1)^{x}(1-z \sqrt{c})^{-2 k_{1}}(1-\bar{w} \sqrt{c})^{-2 k_{2}}(1-c)^{k_{1}+k_{2}} \\
& \times\left(\frac{\sqrt{c}-z}{1-z \sqrt{c}}\right)^{x}\left(1-\frac{(\sqrt{c}-z)(\sqrt{c}-\bar{w})}{(1-z \sqrt{c})(1-\bar{w} \sqrt{c})}\right)^{-2 k_{2}} \frac{1}{\sqrt{2 \pi \Gamma\left(2 k_{1}\right) \Gamma\left(2 k_{2}\right)}} \\
& \times \int_{0}^{\infty} f\left(\rho^{2}\right)\left|\frac{\Gamma\left(k_{2}-k_{1}+\frac{1}{2}+i \rho\right) \Gamma\left(k_{1}+k_{2}-\frac{1}{2}+i \rho\right) \Gamma\left(k_{1}-k_{2}+x+\frac{1}{2}+i \rho\right)}{\Gamma(2 i \rho)}\right| \\
& \times \frac{1}{\Gamma(1+x)}{ }_{2} F_{1}\left(\begin{array}{c}
k_{2}-k_{1}+\frac{1}{2}+i \rho, k_{2}-k_{1}+\frac{1}{2}-i \rho ; \frac{(\sqrt{c}-z)(\sqrt{c}-\bar{w})}{1+x} \\
(1-c)(z \bar{w}-1)
\end{array}\right) d \rho .
\end{aligned}
$$

Now combining expression (4.8) and (4.9), we find a non-symmetric type Poisson kernel for the Meixner functions. Then substituting

$$
\sqrt{\frac{z}{\bar{w}}} \mapsto t, \quad z \bar{w} \mapsto s, \quad x-L \mapsto x, \quad-L \mapsto y,
$$

(and still using $\varepsilon=k_{1}-k_{2}+L$ ) we have

Theorem 4.3. For $x, y \in \mathbb{Z}, \sqrt{|c s|}<|t|<1 / \sqrt{|c s|}, 0<|c|<1$ and $0<|s|<1$ the Meixner functions, as defined in (3.4), satisfy

$$
\begin{aligned}
& \sum_{n=-\infty}^{\infty} \hat{m}_{n}\left(x ;-\frac{1}{2}+i \rho, \varepsilon, c\right) \hat{m}_{n}\left(y ;-\frac{1}{2}+i \rho, \varepsilon, s\right) t^{n}= \\
& \quad t^{y}(1-s)^{2 \varepsilon+y}(1-c)^{2 \varepsilon+y}(1-\sqrt{c s} t)^{-2 \varepsilon-x-y}(\sqrt{s}-\sqrt{c})^{x-y} c^{-\frac{x}{2}} s^{\frac{y}{2}} \frac{\left|\Gamma\left(x+\varepsilon+\frac{1}{2}+i \rho\right)\right|^{2}}{\Gamma(1+x-y)} \\
& \quad \times{ }_{2} F_{1}\left(\begin{array}{c}
-y-\varepsilon+\frac{1}{2}+i \rho,-y-\varepsilon+\frac{1}{2}-i \rho \\
1+x-y
\end{array} ; \frac{(\sqrt{c}-\sqrt{s} t)(\sqrt{c}-\sqrt{s} / t)}{(1-c)(s-1)}\right) .
\end{aligned}
$$

We can consider theorem 4.3 as a non-symmetric type Poisson kernel for the Meixner functions. For $\sqrt{c}$ and $\sqrt{s}$ we use the principal value of the square root.

Remark 4.4. (i) Recall that the Meixner functions can be written in terms of Jacobi functions, which contain the Bessel functions as a limit case [12, §2.3]. In this light we can consider theorem 4.3 as an extension of Graf's addition formula [19, §11.3] for Bessel functions of integer order.

(ii) The conditions on $t$ are exactly the same conditions for the convergence of the generating functions for the Meixner polynomials (4.2) and (4.4).

(iii) The ${ }_{2} F_{1}$-series on the right hand side is the same ${ }_{2} F_{1}$-series as in the Meixner function $\hat{m}_{x}\left(y ;-\frac{1}{2}+i \rho, \varepsilon, \frac{(\sqrt{c}-\sqrt{s} t)(\sqrt{c}-\sqrt{s} / t)}{(1-\sqrt{c s} / t)(1-\sqrt{c s} t)}\right)$.

(iv) Theorem 4.3 holds for all $\rho=\sqrt{y}, y \in \operatorname{supp} \mu$, so the theorem also holds for the values of $\rho$ corresponding to the complementary series representation. For the values of $\rho$ corresponding to the negative discrete series representations, $\rho=i\left(\varepsilon+\frac{1}{2}+j\right)$, the sum terminates at $n=j+1$. So this case gives the non-symmetric Poisson kernel for the Meixner polynomials.

(v) If we put $t=1$ and $s=c \in \mathbb{R}$, we find the dual orthogonality relations (3.7) for the Meixner functions.

(vi) From the substitution $-L \mapsto y$ it is not clear that $y \in \mathbb{Z}$ instead of $-y \in \mathbb{Z}_{\geq 0}$. Using (4.7) and the reflection formula for the $\Gamma$-function, we can show that the right hand side of the 
summation formula is invariant under interchanging both $c$ and $s$, and $x$ and $y$. And then since $x \in \mathbb{Z}$, the same holds for $y$. This also shows that $c$ may be complex valued.

We may also try other realizations. Instead of the element $X_{c}$, we may consider the element $X_{c}^{\phi}$, which is still self-adjoint, defined by, cf. [11],

$$
X_{c}^{\phi}=-\frac{1+c}{2 \sqrt{c}} H+e^{i \phi} B-e^{-i \phi} C, \quad \phi \in[0,2 \pi] .
$$

And we realize the orthonormal basisvectors as Meixner polynomials, as in [11, §2]. This method leads to the summations formula (3.11) of theorem 3.6 for complex values of $c$.

\section{REFERENCES}

[1] N.I. Akhiezer, The classical moment problem and some related questions in analysis, Hafner, 1965.

[2] G.E. Andrews, R. Askey, R. Roy, Special functions, Encycl. Math. Appl. 71, Cambridge Univ. Press, 1999.

[3] A. Erdélyi, W. Magnus, F. Oberhettinger, F.G. Tricomi, Higher transcendental functions, Vol. 1, McGraw-Hill, 1953.

[4] Y.I. Granovskii, A.S. Zhedanov, New construction of 3nj-symbols, J. Phys. A: Math. Gen. 26 (1993), $4339-4344$.

[5] S.C. Hille, Canonical representations, PhD thesis, Leiden, 1999.

[6] M.E.H. Ismail, D. Stanton, Classical Orthogonal Polynomials as Moments, Canad. J. Math. 49 (1997), $520-542$.

[7] G.A. Kerimov, Y.A. Verdiyev, Clebsch-Gordan problem for the three-dimendional Lorentz group in the elliptic basis: II. Tensor products involving discrete series, J. Phys. A: Math. Gen. 32 (1999), 3385-3395.

[8] R. Koekoek and R.F. Swarttouw, The Askey-scheme of hypergeometric orthogonal polynomials and its qanalogue, Report 98-17, Technical University Delft, Delft, 1998; also available from ftp.twi.tudelft.nl in directory /pub/publications/tech-reports.

[9] E. Koelink, Spectral theory and special functions, preprint 2001, math.CA/0107036.

[10] H.T. Koelink, J. Van der Jeugt, Convolutions for orthogonal polynomials from Lie and quantum algebra representations, SIAM J. Math. Anal. 29 (1998), 794-822.

[11] H.T. Koelink, J. Van der Jeugt, Bilinear generating funcions for orthogonal polynomials, Constr. Approx. 14 (1999), 481-497.

[12] T.H. Koornwinder, Jacobi functions and analysis on noncompact semisimple Lie groups, in: Special functions: group theoretical aspects and applications, R.A. Askey, T.H. Koornwinder, W. Schempp (Eds.), D. Reidel Publ. Comp., Dordrecht, 1984, 1-85.

[13] D.R. Masson, J. Repka Spectral theory of Jacobi matrices in $\ell^{2}(\mathbb{Z})$ and the $\mathfrak{s u}(1,1)$ Lie algebra, SIAM J. Math. Anal. 22 (1991), 1131-1146.

[14] B. Ørsted, G. Zhang, $L^{2}$-versions of the Howe correspondence I, Math. Scand. 80 (1997), 125-160.

[15] K. Schmüdgen, Unbounded operator algebras and representation theory, Operator theory, vol. 37, Birkhäuser, 1990.

[16] J. Van der Jeugt, Coupling coefficients for Lie algebra representations and addition formulas for special functions, J. Math. Phys. 38 (1997), 2728-2740.

[17] J. Van der Jeugt, R. Jagannathan, Realizations of $\mathfrak{s u}(1,1)$ and $U_{q}(\mathfrak{s u}(1,1))$ and generating functions for orthogonal polynomials, J. Math. Phys. 39 (1998), 5062-5078.

[18] N.J. Vilenkin, A.U. Klimyk, Representations of Lie groups and special functions, Vol. 1, Kluwer Academic Publishers, Dordrecht, 1991.

[19] G.N. Watson, Theory of Bessel functions, Cambridge Univ. Press, 2nd ed., 1944.

[20] J.A. Wilson, Some hypergeometric orthogonal polynomials, SIAM J. Math. Anal. 11 (1980), 690-701.

Technische Universiteit Delft, ITS-TWA, Postbus 5031, 2600 GA Delft, The Netherlands E-mail address: W.G.M.Groenevelt@its.tudelft.nl, H.T.Koelink@its.tudelft.nl 